\title{
A Ground Work on Optimization Problem Under Multi-Choice Set
}

\author{
Gurupada Maity \\ Department of Applied Mathematics with Oceanology and Computer Programming, Vidyasagar University, Midnapore-721102, West \\ Bengal, India
}

\begin{abstract}
This paper presents a new direction of optimization problem under multi-choice set. In this study, a new concept of multichoice set is introduced and then the optimization problem under multi-choice set is discussed. The usefulness of optimization problem under multi-choice set is presented by considering examples.
\end{abstract}

Keywords: Optimization Problem; Multi-choice Set; Multi-choice Optimization; Transportation Problem; Multi-choice Decision Making

\section{Introduction}

Optimization is the mathematical discipline which is concerned with finding the maximum and minimum of functions with or without constraints. In the study of optimization, basically we need to optimize a real function of $\mathrm{n}$ variables $f\left(x_{1}, x_{2}, \ldots, x_{n}\right)$ with or without constraints. Main aim of this paper is develop and solve Multi-Choice Optimization Problem (MCOP) under multi-choice set.

Firstly, the question comes to mind, what is meant by multichoice optimization problem? In a classical optimization problem, the objective function $f(x)$ consists with the variable $(x)$ and the real constants which are multiplied with the variable $(x)$ If in place of real constants there be a set real numbers multiplied with at least one of the variables, then the objective function becomes a multi-choice objective function and corresponding problem is MCOP.

Again, if at least one constraint contains a multi-choice set in place of real value then the constraint becomes a multichoice constraint.

An optimization problem in which objective function or at least one of the constraints is of multi-choice nature, then it is called a multi-choice optimization problem.

Let us consider an optimization problem as follows:

$$
\text { minimize } f\left(x_{1}, x_{2}\right)=x_{1}^{2}+(1 \text { or } 3 \text { or } 4) x_{2}^{2}
$$$$
\text { subject to } x_{1}+x_{2} \leq 2
$$$$
\text { (2 or } 3) x_{1}+x_{2} \geq 1
$$

This is a multi-choice optimization problem involving multichoice nature in both objective function and constraint.

After defining such problem, someone may confuse about the following things:

- Is there any real field where this type of optimization problem may arise?

- Is it possible to solve MCOP?

- What is the difference between traditional optimization and MCOP?

The study gives the answer positively to the questions and establish the new direction of optimization problem, namely MCOP. In this research work, the concept of MCOP and it's applicability has been incorporated.
The residue of the paper is organized as follows: Section 2 contains literature review regarding this study. Section 3 presents the Formulation of mathematical model of MCOP. Section 4 demonstrates the usefulness of the proposed study compare to traditional optimization problem. Finally, in Section 5, the conclusion and future scope of work are presented related to our consideration.

\section{Literature Review}

Great mathematician Fermat and Lagrange found the calculus-based formulas for identifying optimum values, while Newton and Gauss proposed iterative methods for moving towards an optimum point. The study on "linear programming" for certain optimization cases was given by George B. Dantzig, although much of the theory had been deduced by Leonid Kantorovich in 1939. Dantzig published the Simplex algorithm in 1947, and John von Neumann developed the theory of duality in the same year. Decision making problems like Transportation Problem (TP), facility location problem, inventory problem etc. are optimization based studies. Different types of optimization models are invented to find solution of decision making problems in the mentioned ground. Some useful studies on optimization problems are presented here.

The basic TP was originally developed by Hitchcock [11] and later independently developed by Koopmans [13]. Charnes and Cooper [4] first presented various approaches on the solution of managerial level problems involving multiple conflicting objective functions. Garfinkl and Rao [8] studied on two objective functions by giving high and low priorities to the objective functions. Cadenas and Verdegay [1] solved multi-objective linear programming problem by fuzzy ranking function. Ebrahimnejad [7] worked on uncertainty under different fuzzy environments. Jiménez and Verdegay [12] used the fuzzy uncertainty in solving solid transportation problem. Gao [9] introduced some properties on continuous uncertain measure in his paper. Goal programming (GP) is one of the most useful and well known decision making technique which was introduced by Charnes et al. [5]. A lot of works based on optimization has been done by several researchers such as Chang [3], Liao [15], Lee [14], Gupta and Kumar [10], and many others. 


\section{International Journal of Science and Research (IJSR) \\ ISSN (Online): 2319-7064 \\ Index Copernicus Value (2013): 6.14 | Impact Factor (2014): 5.611}

Many researchers have developed (Chang [3,4], Charnes et al. [5]) studies on multi-choice parameters but they do not introduced the new area of optimization. In this article, main object is to introduce about the new area of optimization, namely MCOP with proper justification.

\section{Formulation of Mathematical Model of MCOP}

Set theory is the base of mathematics. To cover up the reallife problems, we extended the definition of ordinary Set to a Multi-choice Set. We define some definitions and discus by examples.

Definition 3.1( Set): A Set A is a well defined collection of distinct objects. For example $\mathrm{A}=\{1,2,3,4\}$ is a Set containing four elements.

We extended the concept of Set to Multi-choice Set by considering an element of a Set as a Set of elements. In this case, one element of a Set is the element of another Set. We define this type of Set namely Multi-choice Set as follows.

Definition 3.2 (Multi-choice Set): A Set $S$ is said to be a Multi-choice Set, if at least one element of the Set $S$ is not a simple element, it's a Set of elements.

For example, let $\mathrm{S}$ be a Multi-choice Set then $\mathrm{S}=$ $\left\{a_{1}, a_{2}, \ldots, a_{n}\right\}$ where $a_{i} \in S$ implies $a_{i}=\left\{a_{i}^{1}, a_{i}^{2}, \ldots, a_{i}^{r}\right\}$ for all $i=1,2, \ldots, \mathrm{n}$. If $\mathrm{r}=1$ for all $i$ then $\mathrm{S}$ is an ordinary Set.

The study of optimization based on objective function, so let us know what is meant by function on Multi-choice Set.

Definition 3.3 (Function on Multi-choice Set): A function $f(x)$ is called a function on multi-choice set if the outcome of $f$ corresponding to input is a Multi-choice Set.

Consider the function $f\left(x_{1}, x_{2}\right)=x_{1}^{2}+(1$ or 3 or 4$) x_{2}^{2}$;

Expanding the function we get the following result:

$f\left(x_{1}, x_{2}\right)=x_{1}^{2}+1 \cdot x_{2}^{2}$ or $x_{1}^{2}+3 \cdot x_{2}^{2}$ or $x_{1}^{2}+4 x_{2}^{2}$;

which suggests that one input $\left(x_{1}, x_{2}\right)$ has outcome among three possibilities.

Definition 3.3 (Objective function): The function $f$ that is desired to maximize or minimize is known as objective function.

Definition 3.4 (Multi-choice objective function): An objective function $f$ which is defined on multi-choice set (Definition 3.3 type) is called a multi-choice objective function.

Definition 3.5 (Multi-choice constraint): A constraint (restriction in an optimization problem) in which at least any one coefficient is an element of a Multi-choice Set is called a multi-choice constraint.

Definition 3.6 (MCOP): An optimization problem in which either the objective function is a multi-choice objective function or at least one of the constraints is a multi-choice constraint is termed as MCOP.
To illustrate the MCOP, we consider here the commonly used optimization problem, namely Transportation Problem (TP). The mathematical model of TP in sense of ordinary optimization can be described as follows:

Model 1: minimize $\mathrm{Z}=\sum_{\mathrm{i}=1}^{\mathrm{m}} \sum_{\mathrm{j}=1}^{\mathrm{n}} \mathrm{C}_{\mathrm{ij}} \mathrm{x}_{\mathrm{ij}}$,

$$
\begin{array}{ll}
\text { subject to } & \sum_{\mathrm{j}=1}^{\mathrm{n}} \mathrm{x}_{\mathrm{ij}} \leq \mathrm{a}_{\mathrm{i}}(\mathrm{i}=1,2, \ldots, \mathrm{m}), \\
& \sum_{\mathrm{i}=1}^{\mathrm{m}} \mathrm{x}_{\mathrm{ij}} \geq \mathrm{b}_{\mathrm{j}}(\mathrm{j}=1,2, \ldots, \mathrm{n}), \\
& \mathrm{x}_{\mathrm{ij}} \geq 0 \forall i, j .
\end{array}
$$

Here, $\quad x_{i j}(i=1,2, \ldots, m ; j=1,2, \ldots, n)$ is the decision variable which selects the number of goods to be shifted from origin to destination; $\mathrm{C}_{\mathrm{ij}}$ is the cost parameter corresponding to transportation of unit amount of good from $i$-th origin to $j$-th destination. $a_{i}(i=1,2, \ldots, m)$ is the supply at the $i$-th origin and $b_{j}(j=1,2, \ldots, n)$ is the demand quantities at the $\mathrm{j}$-th destination.

Due to presence of more than one route there may be a confusion for selecting the route as they have different costs. Again, due to unstable situation in the market the demand and supply quantity may be multi-choice in nature. Then the mathematical model becomes a TP of MCOP type, whose mathematical model can be described as follows:

Model 1:

minimize $\mathrm{Z}=\sum_{\mathrm{i}=1}^{\mathrm{m}} \sum_{\mathrm{j}=1}^{\mathrm{n}}\left(\mathrm{C}_{\mathrm{ij}}^{1}\right.$ or $\mathrm{C}_{\mathrm{ij}}^{2}$ or $\ldots$ or $\left.\mathrm{C}_{\mathrm{ij}}^{\mathrm{r}}\right) \mathrm{x}_{\mathrm{ij}}$, subject to $\quad \sum_{j=1}^{n} x_{i j} \leq\left(a_{i}^{1}\right.$ or $a_{i}^{2}$ or $\ldots$ or $\left.a_{i}^{p}\right)$ $(\mathrm{i}=1,2, \ldots, \mathrm{m})$

$$
\sum_{i=1}^{m} x_{i j} \geq b_{j}^{1} \text { or } b_{j}^{2} \text { or } \ldots \text { or } b_{j}^{q}(j=
$$

$1,2, \ldots, \mathrm{n}$

$$
\mathrm{x}_{\mathrm{ij}} \geq 0 \forall i, j .
$$

Reader may think why we think about this type of optimization problem? So let us illustrate the utility of MCOP in compare to traditional optimization Problem.

\section{Utility of MCOP}

The justify the utility of MCOP, we consider some examples of optimization problems.

Example 1. minimize $\mathrm{z}=2 \mathrm{x}_{11}+\mathrm{x}_{12}+3 \mathrm{x}_{21}+\mathrm{x}_{22}$

$$
\text { subject to } \left.\begin{array}{r}
\mathrm{x}_{11}+\mathrm{x}_{12} \leq 10 \\
\mathrm{x}_{21}+\mathrm{x}_{22} \leq 12 \\
\mathrm{x}_{11}+\mathrm{x}_{21} \geq 11 \\
\mathrm{x}_{12}+\mathrm{x}_{22} \geq 11 \\
\mathrm{x}_{\mathrm{ij}} \geq 0 \quad \forall i, j
\end{array}\right\}
$$

The optimal solution is $\min \mathrm{z}=34 ; \mathrm{x}_{11}=10, \mathrm{x}_{12}=0, \mathrm{x}_{21}=$ $1, \mathrm{x}_{22}=11$.

Example 2. minimize $\mathrm{z}=4 \mathrm{x}_{11}+3 \mathrm{x}_{12}+3 \mathrm{x}_{21}+\mathrm{x}_{22}$ subject to (A)

The optimal solution is $\min \mathrm{z}=54 ; \mathrm{x}_{11}=10, \mathrm{x}_{12}=0, \mathrm{x}_{21}=$ $1, \mathrm{x}_{22}=11$.

Example 3. minimize $z=2 x_{11}+x_{12}+3 x_{21}+1 x_{22}$ subject to (A)

The optimal solution is $\min \mathrm{z}=44 ; \mathrm{x}_{11}=0, \mathrm{x}_{12}=10, \mathrm{x}_{21}=$ $11, \mathrm{x}_{22}=1$. 


\section{International Journal of Science and Research (IJSR) \\ ISSN (Online): 2319-7064}

Index Copernicus Value (2013): 6.14 | Impact Factor (2014): 5.611

Example 4. minimize $\mathrm{z}=2 \mathrm{x}_{11}+3 \mathrm{x}_{12}+3 \mathrm{x}_{21}+\mathrm{x}_{22}$ subject to (A)

The optimal solution is $\min \mathrm{z}=34 ; \mathrm{x}_{11}=10, \mathrm{x}_{12}=0, \mathrm{x}_{21}=$ $1, x_{22}=11$.

From the four examples, the following MCOP can be formed.

Example 5. minimize $\mathrm{z}=\{2$ or 4$\} \mathrm{x}_{11}+\{1$ or 3$\} \mathrm{x}_{12}+$ $3 \mathrm{x}_{21}+\mathrm{x}_{22}$ subject to (A)

The study of traditional operations research answered that the optimal solution for minimization problem is obtained by the minimum value of the Multi-choice Set elements and the present case (Example 5) gives optimal solution corresponding to the solution of Example 1. Now if someone ask solve the problem in Example 5 to get the value of $\mathrm{z}$ around 40, then which one among the four Examples 1,2,3 and 4 will produced the optimal solution. The traditional optimization methods fail to establish the result and the study of MCOP is used to solve the problem.

Solution Procedure: In real-life problems if the situation occurs then, we formulate MCOP by considering every possibility, then the multi-choice coefficients are reduced to single coefficients by using binary variables which produces the simple optimization problem and solving the problem we get the optimal solution as well as the optimal selection among the multi-choice coefficients. The Example 5 reduces in the following from:

$\min =\mathrm{d}$;

$\mathrm{z}=(2 * \mathrm{z} 1+(1-\mathrm{z} 1) * 4) * \mathrm{x} 11+(1 * \mathrm{z} 2+(1-\mathrm{z} 2) * 3) * \mathrm{x} 12+3$

$* \times 21+\times 22$

$\mathrm{d}=\mathrm{z}-40$;

$\mathrm{x} 11+\mathrm{x} 12<=10$

$\mathrm{x} 21+\mathrm{x} 22<=12$

$\mathrm{x} 11+\mathrm{x} 21>=11$

$\mathrm{x} 12+\mathrm{x} 22>=11$

$\mathrm{x} 11>=0 ; \mathrm{x} 12>=0 ; \mathrm{x} 21>=0 ; \times 22>=0$;

@bin(z1);@bin(z2);

$\mathrm{d}>=0$;

end

Optimal solution is $\mathrm{x} 11=8, \times 12=2, \mathrm{x} 21=3, \mathrm{x} 22=9$; choices are 2 from $\{2,4\} ; 3$ from $\{1,3\}$. So the solution suggests that the better solution is produced by Example 4 .

Hence we are in a situation in which the traditional optimization problem fails to solve and give conclusion for optimal selection among a set of multi-choice options, but the Study of MCOP nicely concluded the proposed query. When there are several routes of transportation in a real-life $\mathrm{TP}$, then the mathematical model takes the form of MCOP, so a lot of optimization problem belongs to the class of MCOP.

\section{Conclusion and Future Scope of Research}

In this paper, we have introduced the new area of optimization problem namely MCOP. We have proposed some useful definitions to know about MCOP. The utility of MCOP is presented nicely with the help of numerical examples. MCOP is found in many real-life decision making situations like transportation problem, inventory, route selection problem etc. The concept of MCOP can give a better selection way of optimal strategy among a class of strategies in decision making problems.

There are many directions for future study. One main direction is to design and extend the proposed study to a class of MCOP, where uncertain coefficients are presented in both objective functions and constraints. Another direction is that this study can be implemented to find the solution of managerial problems occurred in the field of multi-objective transportation problem, supply chain management, inventory, etc. when there are multi-choice routes of transportation.

\section{Acknowledgment}

The author is highly acknowledged to the University Grants Commission of India for supporting the financial assistance to continue this research work under JRF(UGC) scheme Sanction letter number [F.17-130/1998(SA-I)] dated 26/06/2014.

\section{References}

[1] Cadenas, J.M. and Verdegay, JL, Using ranking functions in multi-objective fuzzy linear programming, Fuzzy sets and systems 111 (1), 47-53, 2000

[2] Chang, C.T., Multi-choice goal programming, Omega: 35, 389-396, 2007

[3] Chang, C.T., Revised multi-choice goal programming, Applied Mathematical Modelling: 32, 2587-2595, 2008.

[4] Charnes, A., Cooper, W.W., Management models and industrial applications of linear programming. Management Science, 4(1), 38-91, 1957.

[5] Charnes, A., Cooper, W.W. and Ferguson, R.O., Optimal estimation of executive compensation by linear programming, Management Science: 1, 138-151, 1955.

[6] Delgado, M., Verdegay , J. L. and Vila, MA.,A general model for fuzzy linear programming, Fuzzy Sets and systems 29 (1), 21-29, 1989.

[7] Ebrahimnejad, A., A simplified new approach for solving fuzzy transportation problems with generalized trapezoidal fuzzy numbers, Applied Soft Computing, 19, 171-176, 2014.

[8] Garfinkl, R.S., Rao, M.R., The bottleneck transportation problem. Naval Research Logistics Quarterly, 18, $465-$ $472,1971$.

[9] Gao X., Some Properties of Continuous Uncertain Measure, International Journal of Uncertainty, Fuzziness and Knowledge-Based Systems, 17, 419-426, 2009.

[10] Gupta, A. and Kumar, A., A new method for solving linear multi-objective transportation problems with fuzzy parameters. Applied Mathematical Modelling, 36, 1421-1430, (2012).

[11] Hitchcock, F.L., The distribution of a product from several sources to numerous localities, Journal of Math. Phys: 20, 224-230, 1941.

[12] Jiménez, F. and Verdegay, JL., Solving fuzzy solid transportation problems by an evolutionary algorithm based parametric approach, European Journal of Operational Research 117 (3), 485-510, 1999. 


\section{International Journal of Science and Research (IJSR) \\ ISSN (Online): 2319-7064}

Index Copernicus Value (2013): 6.14 | Impact Factor (2014): 5.611

[13] Koopmans, T.C., Optimum utilization of the transportation System, Econometrica: 17, 3-4, 1949.

[14]Lee, S.M., Goal programming for decision analysis, Auerbach Publishers: Philadelphia, 1972.

[15]Liao, C.N., Formulating the multi-segment goal programming, Computers \& Industrial Engineering: 56(1), 138-141, 2009.

[16]Liu B., Uncertainty Theory, 2nd ed., Springer-Verlag, Berlin, 2007. 